\title{
Sub-diffraction imaging on standard microscopes through photobleaching microscopy with non- linear processing
}

\author{
Sebastian Munck ${ }^{1,2,3}$, Katarzyna Miskiewicz ${ }^{1,2}$, Ragna Sannerud ${ }^{1,2}$, Silvia A. Menchon ${ }^{1,2}$, Liya Jose ${ }^{1,2}$, \\ Rainer Heintzmann ${ }^{4,5,6, *}$, Patrik Verstreken ${ }^{1,2, *}$ and Wim Annaert ${ }^{1,2, *, \pm}$ \\ ${ }^{1}$ VIB Center for the Biology of Disease, Herestraat 49, Bus 602, 3000 Leuven, Belgium \\ ${ }^{2} \mathrm{~K}$. U. Leuven, Center for Human Genetics, Herestraat 49, Bus 602, 3000 Leuven, Belgium \\ ${ }^{3}$ VIB, LiMoNe, Herestraat 49, Bus 602, 3000 Leuven, Belgium \\ ${ }^{4}$ Institute of Physical Chemistry, Friedrich-Schiller University Jena, Helmholtzweg 4, 07743 Jena, Germany \\ ${ }^{5}$ Institute of Photonic Technology, Albert-Einstein Str. 9, 07745 Jena, Germany \\ ${ }^{6}$ Randall Division of Cell and Molecular Biophysics, King's College London, NHH, Guy's Campus, London, SE1 1UL, UK \\ ${ }_{\ddagger}^{*}$ These authors contributed equally to this work
}

\begin{abstract}
Summary
Visualization of organelles and molecules at nanometer resolution is revolutionizing the biological sciences. However, such technology is still limited for many cell biologists. We present here a novel approach using photobleaching microscopy with non-linear processing (PiMP) for sub-diffraction imaging. Bleaching of fluorophores both within the single-molecule regime and beyond allows visualization of stochastic representations of sub-populations of fluorophores by imaging the same region over time. Our method is based on enhancing the probable positions of the fluorophores underlying the images. The random nature of the bleached fluorophores is assessed by calculating the deviation of the local actual bleached fluorescence intensity to the average bleach expectation as given by the overall decay of intensity. Subtracting measured from estimated decay images yields differential images. Non-linear enhancement of maxima in these diffraction-limited differential images approximates the positions of the underlying structure. Summing many such processed differential images yields a super-resolution PiMP image. PiMP allows multi-color, three-dimensional sub-diffraction imaging of cells and tissues using common fluorophores and can be implemented on standard wide-field or confocal systems.
\end{abstract}

Key words: Bleaching, Super-resolution microscopy, Method, PiMP

\section{Introduction}

Numerous super-resolution imaging technologies have recently emerged, including stimulated emission depletion (STED), photoactivated localization microscopy (PALM) and stochastic optical reconstruction microscopy (STORM) (Hell and Wichmann, 1994; Gustafsson et al., 1999; Gustafsson, 2000; Heintzmann et al., 2002; Betzig et al., 2006; Hess et al., 2006; Rust et al., 2006; Fölling et al., 2008; Heilemann et al., 2008; Dertinger et al., 2009; Gong et al., 2010). These technologies allow for the accurate imaging of labeled molecules by separating their emission in space or time, enabling researchers to bypass the resolution limit of conventional light microscopy (Abbe, 1873). Given the impact on biological research, super-resolution technology has gained great attention; however, assembling a super-resolution device or acquiring an integrated solution can be expensive and custom-built systems still outperform the commercial ones. Moreover, the aforementioned strategies are not applicable without additional hardware and/or imaging sensitivity. A different strategy that allows easy accessible super-resolution imaging is thus a very attractive alternative.

Here we describe a novel approach to achieve sub-diffraction imaging beyond the point where emitters are in such close proximity that they form a single indiscernible diffraction pattern as described by the Abbe limit (Abbe, 1873). We exploit stochastic processes to generate multiple consecutive subimages that can be reconstructed to yield a significantly improved image at sub-diffraction resolution. More specifically, we use bleaching to image sub-populations of fluorophores, hence the name 'photobleaching microscopy with non-linear processing' or PiMP. Pointillistic approaches (such as PALM and STORM) exploit the principle of decomposing the object over multiple images, but require only a small subpopulation of isolated single molecules to be present per frame. In contrast to these, we analyze the positions at which the bleaching deviates from the expected (average) bleaching per frame. Such analyses are possible because the number of bleached fluorophores on densely labeled neighboring structures (that might be located at a distance below the diffraction limit) is in most cases not equal owing to the stochastic nature of bleaching. This stochastic effect can be used beyond the singlemolecule regime to approximate probable fluorophore locations in the underlying structure. Hence, the combination of extracting and enhancing spatial information of sub-populations of fluorophores from differential images can yield a subdiffraction image revealing information otherwise concealed by diffraction. Biological samples usually exhibit a redundancy of labeling with respect to the underlying structural information 
even at high resolution. The redundancy in labeling allows analysis of images using PiMP with typical bleach-rates of multiple fluorophores per frame and per position in the object (i.e. per final resolution element in the image). This redundancy, together with the stochastic decay of labeling intensity over time, allows the retrieval of significantly more structural detail than possible in conventional microscopic imaging, where a single image is used to reveal 'all' fluorophores at the same time.

We have tested multiple fluorophore arrangements and acquisition parameters using computer-based simulations and provide several examples of PiMP imaging of biological samples using both confocal and wide-field microscopes. Moreover, we compare the images achievable with PiMP with established super-resolution methods. Given that PiMP is compatible with ordinary fluorescence and confocal microscopes, allowing multicolor and 3D imaging, our strategy now makes subdiffraction imaging easily accessible for many cell biologists.

\section{Results}

\section{Establishing PiMP}

In conventional fluorescence microscopy, a single image is typically acquired to reveal the image of the labeled object. Owing to diffraction, an image of, for example, a dense point grid
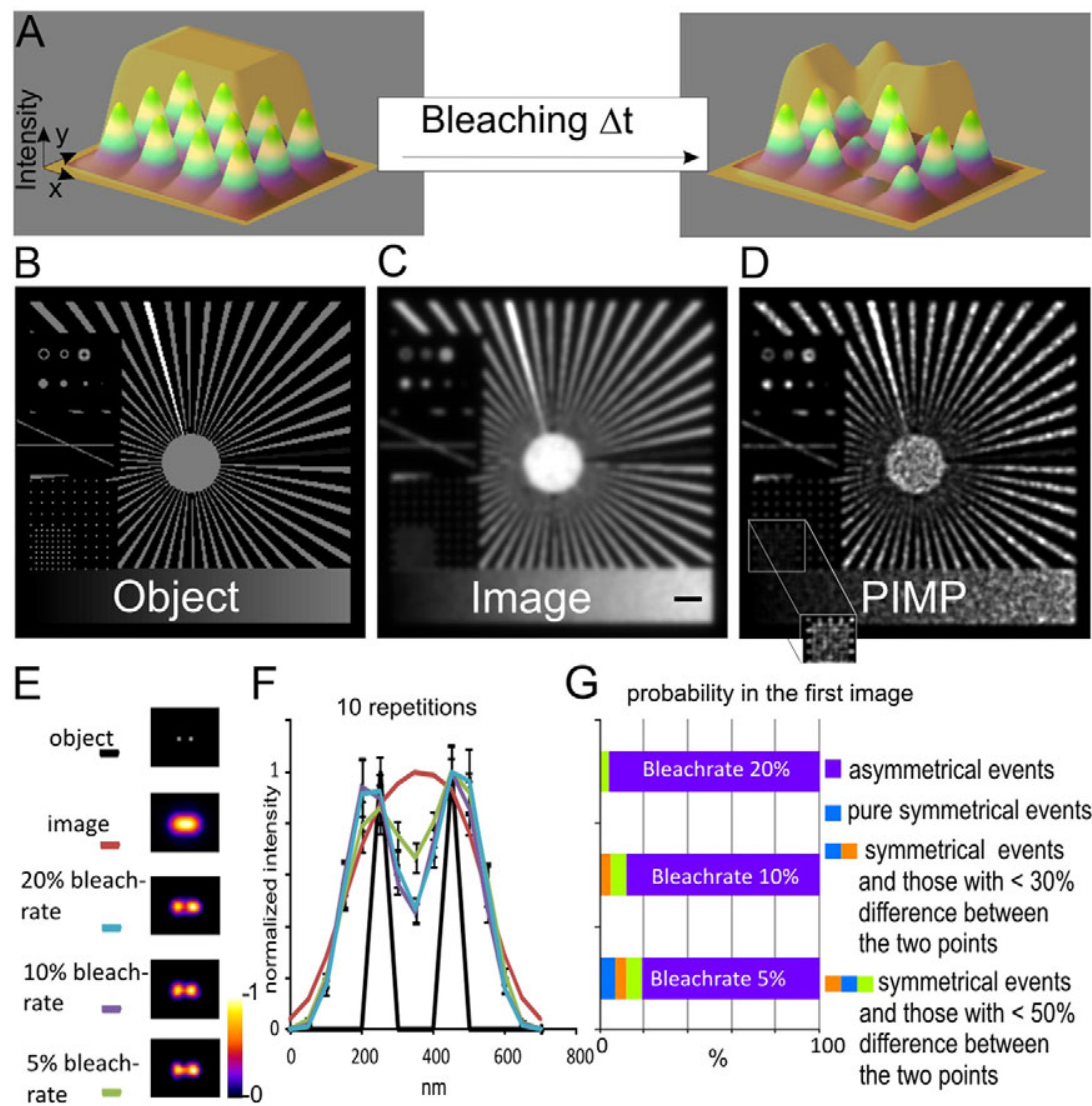

$\mathrm{F}$

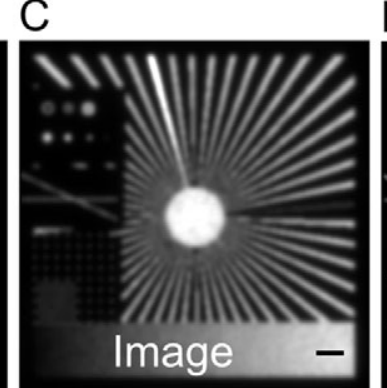

D

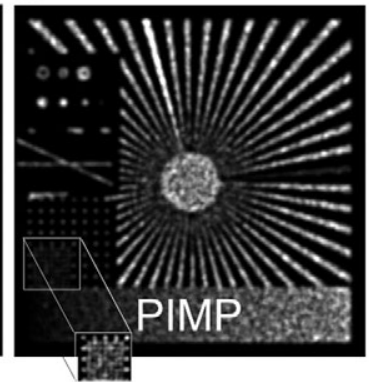

$G$ probability in the first image
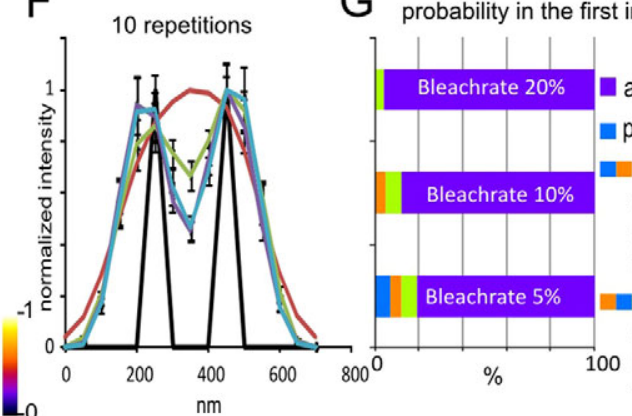

asymmetrical events

pure symmetrical events

III symmetrical events and those with $<30 \%$ difference between the two points

symmetrical events and those with $<50 \%$

rate $=\mathrm{nm}$ the two points

H \begin{tabular}{|c|c|c|c|c|c|}
\hline $\begin{array}{c}\text { Number of } \\
\text { images }\end{array}$ & Dye labeling & $\begin{array}{c}\text { Bleaching } \\
\text { probability }\end{array}$ & $\begin{array}{c}\text { Number of } \\
\text { photons }\end{array}$ & $\begin{array}{c}\text { Filter size }(\sigma \text { of 1st filter 2nd } \\
\text { being double in size) }\end{array}$ & $\begin{array}{c}\text { Gaussian smoothing } \\
\text { in the time direction }\end{array}$ \\
\hline 100 & $\begin{array}{c}50 \text { dyes } / 50 \times 50 \mathrm{~nm} \\
\text { region }\end{array}$ & $5 \%$ & 1000 & 1.1 & 0.8 \\
\hline
\end{tabular}

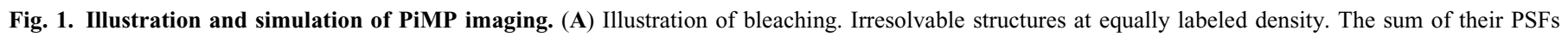

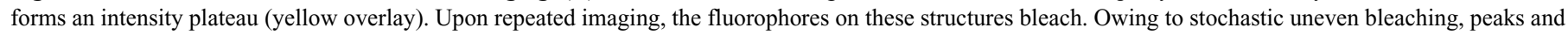
troughs of different heights appear in the 'yellow plateau'. Simulation of PiMP using a simulated wide-field PSF. For the calculation, we assumed an

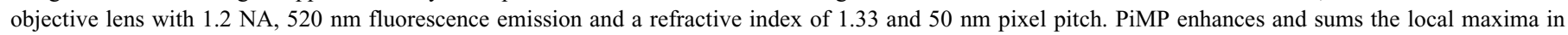

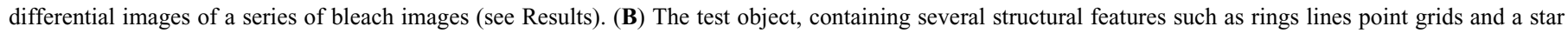

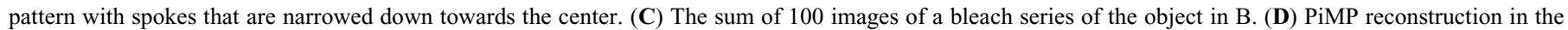
presence of photon noise (insert shows changed contrast). Scale bar: $1 \mu \mathrm{m}$. In contrast to single-molecule techniques, PiMP does not need the unambiguous identification of single molecules. This leads to events where neighboring structures bleach at the same time. (E) To explain the image formation and the

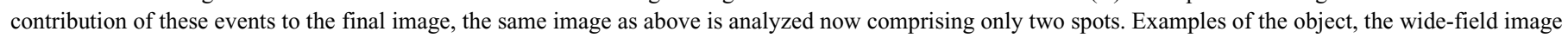

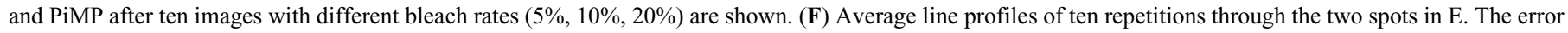

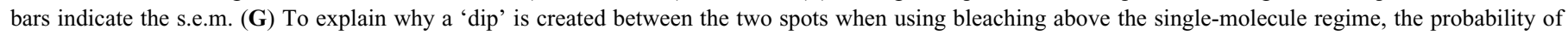

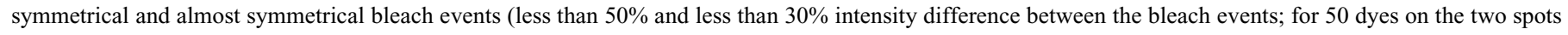

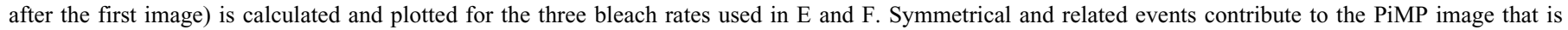
generated, but owing to their minor stochastic contribution to the final image, allow separating the two points as separated entities. (H) Table displaying the optimized simulation and reconstruction parameters used in D, with variations of these parameters shown in supplementary material Figs S2-S4. 
(spaced closer than the resolution limit) will show a plateau of uniform brightness (Fig. 1A). However, by acquiring consecutive images of the same region, inhomogeneity in each image is revealed, caused mostly by stochastical fluorophore bleaching, but also by other brightness fluctuations such as blinking or photoswitching. During bleaching, the image exhibits peaks and troughs because of non-uniform bleaching of fluorophores (Fig. 1A). This stochastic process changes the visible population of fluorophores. The ability to separate subpopulations of fluorophores in consecutive images provides more positional information and allows us to extract structural information beyond the diffraction limit. PiMP analyzes these changes in image brightness over typically tens to hundreds of images, with the aim of revealing the underlying structure of the emitters.

To provide evidence for this idea, we modeled PiMP in silico using a complex object (Fig. 1B) and tested the system under several simulated conditions. The test object accommodated many different features to assess the resolution and detect potential artifacts of the technology. The center part of our test object refers to a general optical instrument test pattern akin to the 'Siemens star' or ISO (International Organization for Standardization) 15775 test pattern. In our case, the pattern was sampled with a $50 \mathrm{~nm}$ pixel size and consists of 50 radial spokes scaling in size with distance from the center. The object was labeled homogenously with 50 dye molecules per pixel in the spokes with one of these spokes kept at double and one at $20 \%$ of fluorophores per pixel. The spokes meet at a homogenous round object in the center with the same labeling concentration. The test object further contains disks and rings of different sizes, one of them having smooth borders (rounded to the nearest integer number of molecules) to investigate the performance of the algorithm for objects with less confined labeling density at its borders. Moreover, it contains two lines crossing at an angle of $25^{\circ}$ and additionally an array of points spaced at distances larger $(400 \mathrm{~nm})$ and smaller $(200 \mathrm{~nm})$ than Abbe's resolution limit $\left(D_{\min }=\lambda / 2 \mathrm{NA}=520 / 2.4=217 \mathrm{~nm}\right)$. Each of these points contains 100 fluorophores. In the lower part of the object, we placed a linear concentration gradient to test for overall brightness linearity of the method.

Next, we simulated the bleaching events of fluorophores during the acquisition of a series of images of this test pattern and by assuming a constant average bleaching rate of fluorophores located on each of the pixels of the object. For each individual fluorophore remaining in a frame, we determined whether it bleached (probability $\left.P_{\text {bleach }}\right)$ during this frame $(t, t+\tau)$, with frametime $t$ and frame integration time $\tau$. Subsequently, each image of the obtained sequence of remaining fluorophores was imaged by convolution with the point spread function (PSF, $520 \mathrm{~nm}, 1.2 \mathrm{NA}$ ) and subjected to Poisson noise (with the expected number of detected photons assumed as 1000 per molecule, if not stated otherwise). Upon regular imaging, the dense array of points is converted to a homogenous plateau and the rings are filled and the lines blurred (compare left inset in Fig. 1B,C). The spokes of the test pattern became indistinguishable near the center (Fig. 1C). Bleaching images of the test sample are shown in supplementary material Fig. S1AC, with a bleaching probability of $P_{\text {bleach }}=5 \%$. The areas affected by bleaching (more or less than average) were assessed by calculating the absolute difference of an image from the previous image dimmed by the bleaching per frame. These differential images were then used to estimate the positions of fluorophores. To estimate the average bleaching decay of intensity for each image, we calculated the overall brightness ratio $\alpha_{n}$ of each image $\left(I_{n}\right)$ to its preceding image. By multiplying the preceding image with $\alpha_{n}$, we created the expected bleached image. Finally, the difference of these expected bleach images to each image yielded the differential images $\left(D_{n}\right): D_{n}(x, y)=\left|\alpha_{n} I_{n}-I_{n+1}\right|$ (supplementary material Fig. S1D-F). The probability for a fluorophore to bleach during a frame is given as $P_{\text {bleach }}=1-\alpha$. The differential images $D_{n}$ were still diffraction limited, but their (bleach) maxima corresponded to a randomized sub-population of the fluorophores that were present on the object. As such, they contained less information than the raw starting images because fewer fluorophores have contributed to them. However, because these sub-images were less 'crowded', non-linear image processing could enhance these positions as selected by bleach events. Thus the combination of randomized stochastic selection and non-linear image processing extends the detection pass-band of the system beyond Abbe's limit (see the Materials and Methods).

In this work, we used a two-step process for defining the nonlinear filter (filter). First, we used a band-pass Mexican hat filter, akin to unsharp masking in Photoshop, consisting of subtracting two Gaussian-blurred versions with different sizes of the filter kernel. This Mexican hat filtering was followed by a Heaviside step function, where negative values were set to zero. The filter was used to enhance the peaks in the differential images $\left(D_{n}\right) \quad\left[I_{\text {filtered }}=\sum\right.$ filter $\left.\left(D_{n}\right)\right]$. Similar strategies, for example to enhance speckles, have been used earlier in dynamic speckle illumination microscopy with wavelet prefiltering (Ventalon et al., 2007).

Setting negative values to zero as well as taking the absolute value to obtain $D_{n}$ are the main contributions to the non-linearity in PiMP processing. Furthermore, empirically, we noticed that the sensitivity of the filtered differential images to photon noise could be further reduced by applying a weak ( $\sigma=0.8$ frames) Gaussian filter along time before the calculation of the differential images (see the Materials and Methods). The sum of the filtered differential images now generated a super-resolved image; however, the brightness was not preserved linearly when compared with the starting image. This is because the differential images provided an estimate of the local deviation from the decay of intensity. Such a deviation was expected to be on average proportional to the square root of the number contributing molecules (which can be estimated from the sum of all raw images). We therefore corrected for the brightness by multiplying the result of the summed image with the square root of all summed images from the bleach-series resulting in the final brightness-corrected PiMP image $\left(I_{\text {PiMP }}=I_{\text {filtered }} \sqrt{\sum_{n} I_{n}}\right)$. When the simulated images were generated using PiMP, more details became visible compared with the conventional image; for example, at the spokes near the center (Fig. 1D) and in the array of spots (lower left corner in Fig. 1D). The bright and the dark spokes of the test pattern also remained visible with PiMP.

Because PiMP operates beyond the single-molecule domain and uses the approximated position of fluctuating fluorophores, a potential concern is that artifactual points between structures could be generated as a result of simultaneous and uniform 
bleaching of two adjacent structures (leading to symmetrical profiles thus called symmetrical bleach events). Therefore, to test this, we simulated two closely located objects (i.e. two adjacent points as also included in the unresolvable grid in the test pattern above). We first compared different bleach rates (5\%, 10\% and $20 \%$ ) as a basis to generate a respective PiMP image. Fig. 1E shows examples of the in silico object, wide-field image (50 fluorophores per point) and the consecutive PiMP result (reconstructed from ten differential images each). In contrast to the wide-field image, the PiMP images exhibited a trough between the two objects allowing us to discriminate the disjointed nature of the object. Next, we repeated this experiment ten times and generated an average line profile (Fig. 1F) through the two points. It can be seen that PiMP separates the two points reliably and that no artifactual points are generated.

The reason for this is the fact that PiMP sums the individual detected events in the individual frames by preserving their relative brightness. In the case where both structures bleached symmetrically at a rate above or below the expected bleach rate, this led to some signal being detected in the middle (as well as at the real location of the points). However, the probability and thus the occurrence for such symmetrical events was less than for asymmetrical events (that led to only one of the points being highlighted). Therefore, these events contribute little to the final image. This is illustrated in Fig. $1 \mathrm{G}$, where we calculated the probability for symmetrical and asymmetrical bleach events between the two points for the three bleach rates used in Fig. 1E,F for the first image. To also accommodate 'almost' (or quasi) symmetrical events (such as eight bleach events on one point and nine on the other point), which will have a similar effect as symmetrical events, we examined symmetrical events and those events that showed less than $30 \%$ and less than $50 \%$ brightness difference between the two points. To judge the contribution to the PiMP image, we plotted the differential probabilities for the first image generated (Fig. 1G). Clearly asymmetrical bleach events outweigh the symmetrical or closeto-symmetrical ones in all cases. Because the PiMP reconstruction is based on more than one image, we could estimate that the probability to constantly obtain images with symmetrical (and related) bleach events decreased dramatically with multiple images used (when assuming the probability of the first image upon repetition, the probability decreased exponentially). The graph in Fig. $1 \mathrm{G}$ illustrates the ability to separate two points using stochastic events beyond the single molecule regime, indicating that the likelihood of artifactual points being generated between structures is very low.

We optimized our approach using the complex test image and varying parameters such as the number of frames for the processing, bleach-rates and dye labeling density (supplementary material Fig. S2). In addition, we also have explored low- and high-sensitivity acquisition and investigated how this affects PiMP processing (supplementary material Fig. S3). Finally, we compared the PiMP processing with top-hat filtering only and with superresolution optical fluctuation imaging SOFI (Dertinger et al., 2009), showing improved image linearity using PiMP (supplementary material Fig. S3). Moreover, we tested how filter sizes and linear and non-linear deconvolution strategies affect PiMP processing and included a comparison with linear and nonlinear deconvolution. These experiments consistently indicate that more detail of the original object was visible with PiMP (supplementary material Fig. S4). The interpretation of the different parameters used in supplementary material Figs S2-S4 resulted in a set of optimized parameters for PiMP, which were used in subsequent experiments (Fig. 1H), unless stated otherwise.

We first demonstrated the applicability of our approach using imaging of fluorescent beads and provide evidence that, compared with confocal imaging, we can achieve significantly better separation of two closely localized beads using PiMP (supplementary material Fig. S5). Indeed, PiMP permits us to clearly separate neighboring beads much better and also resolves beads whose fluorescence distributions were indistinguishable by confocal microscopy. In conclusion, these experiments indicate that PiMP enables super-resolution on simulated and physical test objects.

\section{PiMP on biological samples}

To investigate the performance of PiMP on biological samples, we immunolabeled microtubules with Alexa Fluor 488 secondary antibodies in mouse embryonic fibroblasts (MEFs) (Fig. 2A). When cells were scanned 25 times with $488 \mathrm{~nm}$ light using a wide-field microscope, progressive bleaching of the sample was observed. Imaging using PiMP reveals labeled microtubules with a higher resolution compared with a regular wide-field image.

To validate the reproducibility of PiMP reconstruction using the same sample and testing for the stochastic variability of the reconstruction, we compared the PiMP analysis derived from images 0-25 (Fig. 2B) with the PiMP analysis obtained from the following 26-50 frames (Fig. 2C). This demonstrates that both PiMP images are comparable with slightly more noise present in the second reconstruction, which is due to the progressed bleaching in the second series of images. This highlights the consistency of PiMP analysis in biological samples.

We compared PiMP with well-established superresolution techniques. For this purpose, microtubules were coimmunolabeled with another secondary antibody, now conjugated to Alexa Fluor 568. We used a dSTORM approach to image the Alexa Fluor 568 dual-labeled microtubules in the presence of thiols and using $405 \mathrm{~nm}$ light to aid the return of fluorophores to their ground state (Fölling et al., 2008; Heilemann et al., 2008; Heilemann et al., 2009; van de Linde et al., 2011) (Fig. 2D). Compared with PiMP analysis (Fig. 2B), both approaches revealed the same structures (quantified in Fig. 2G), with dSTORM achieving better resolution as revealed by a smaller footprint of the microtubular width and a slightly more spotted appearance compared with PiMP. However, in dSTORM we needed to analyze many more images to reveal the microtubular structure $(20,000)$ compared with PiMP (25).

To further compare and test our approach, we imaged the same preparation (but a different region) on a commercially available structured illumination (SIM) set-up. Dual-color N-SIM imaging (using a 1.49 NA lens) improved the resolution when compared with the wide-field image (Fig. 2E,F). The comparison of N-SIM (green channel) performance with PiMP (Fig. 2B) indicated that despite using a less effective objective lens (NA 1.3), PiMP resulted in a slightly smaller footprint of microtubule width (Fig. 2G). Overall, these experiments highlight that the subdiffraction performance of PiMP is within the range as achieved by established methods such as ASTORM and SIM. Moreover, because of the stochasticity of antibody binding, both dual color PiMP (Fig. 2H,I) as well as SIM (Fig. 2E,F) yielded a similar 

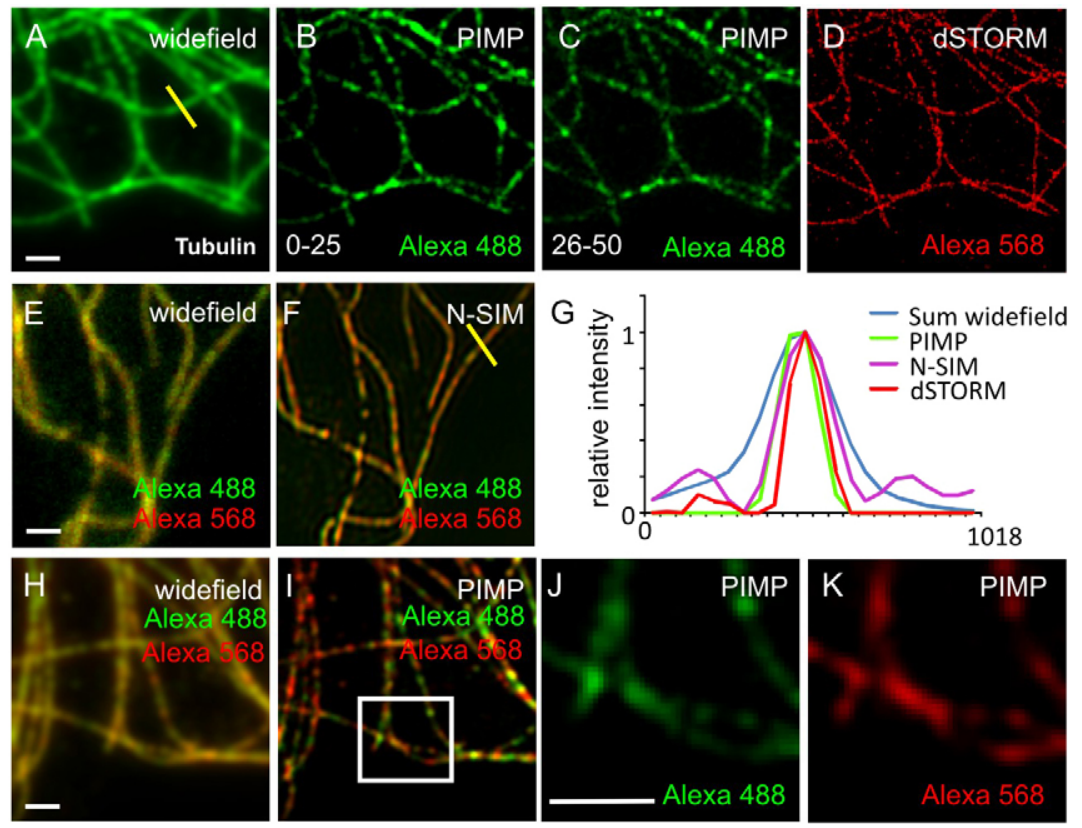

Fig. 2. Control experiments and comparison of PiMP on biological specimens with existing nanoscopical techniques. (A) Wide-field microscopy of microtubules immunolabeled with anti-tubulin and visualized using Alexa-Fluor-488-conjugated secondary antibodies. Sum of 25 sequential frames. (B) PiMP processing of the series used in A yields improved resolution. (C) PiMP reconstructions of the same region as used in A. The second reconstruction is made from the consecutive images 26-50. B and C reveal the same structures with slightly more noise in the PiMP image calculated from the series consisting of the images 26-50 because of progressed bleaching of the sample. B and C demonstrate the reproducibility of PiMP imaging. (D) dSTORM image of the same microtubules as in A and B revealed by Alexa-Fluor-568-conjugated secondary antibodies. The dSTORM reconstruction of microtubules at $30 \mathrm{~nm}$ was achieved using the QuickPALM plugin (Henriques et al., 2010). (E) Wide-field and (F) N-SIM image of the microtubule sample labeled with Alexa-Fluor-488- and Alexa-Fluor-568-conjugated secondary antibodies (same coverslip as A-C). Image in F reveals the microtubules patterned in the two colors as a result of the stochastic binding of antibodies. (G) Quantification of the microtubule width as measured in A, B and C (region indicated by the yellow line in A) and of the green channel of the N-SIM image (F; indicated by the yellow line). (H) Wide-field and (I) PiMP images of the same sample (different cell) revealing the same type of patterning as in E,F.

$(\mathbf{J}, \mathbf{K})$ Zoomed images of a relative homogenous region of I (indicated by the white square); representing that principally the same structures are revealed when PiMP is applied on the same structure using different antibodies, underscoring the reliability of PiMP. Details on imaging conditions are given in supplementary material Table S1. Scale bars: $1 \mu \mathrm{m}$.

patterned appearance for the two colors on the microtubules (Fig. 2F,I). This patterned appearance was visible in wide-field image, but became far more prominent with sub-diffraction imaging. When analyzing regions of relative homogenous staining, PiMP reconstruction displays the same structure in both color channels (Fig. 2J,K).

To further scrutinize the versatile use of PiMP, we imaged the Drosophila neuromuscular junction (NMJ) in larval tissue using a confocal microscope. Bruchpilot (BRP) is an integral component of the active-zone where synaptic vesicles fuse with the membrane and the protein organizes in a 'parasol-like' structure [which appears as a 'T-bar' in transmission electron microscopy (TEM)] with its C-terminal end in the cytoplasm (Kittel et al., 2006). PiMP resolves clearly the ring-like appearance of the immunolabeled $\mathrm{C}$ terminal BRP antigen that had earlier been revealed with superresolution STED microscopy (Kittel et al., 2006; Fouquet et al., 2009) (Fig. 3A,B). In addition, the inner ring diameter measured on PiMP images closely fits to the size of the T-bar as measured by TEM (Fig. 3C,D) (171.1 $\pm 17.3 \mathrm{~nm}, n=16$ for PiMP and $145.3 \pm 8.6 \mathrm{~nm}, n=18$ for TEM). The TEM images acquired from conventionally prepared samples and standard exposures to the electron beam can be subject to some level of distortion. It is expected that this leads to slight shrinking of the imaged structure and therefore to an underestimation in our comparison. Nonetheless, our BRP images and T-bar sizes obtained by PiMP imaging were very similar to those obtained by STED (and TEM) microscopy earlier (Kittel et al., 2006; Fouquet et al., 2009; Hallermann et al., 2010), further underscoring the reliability of PiMP. These results additionally demonstrate that the full capacity of optical sectioning of a confocal microscope can be used for subdiffraction imaging, which allows PiMP analysis in tissues.

To test whether PiMP could be used to increase resolution in $z$-stacks, we again turned to immunolabeled microtubules. When samples are imaged at different $z$-positions additional 'unrecorded' bleaching occurs. It is clear that the unrecorded bleaching and consequent loss of signal poses a potential limit to imaging of large $z$-stacks with PiMP. To allow PiMP imaging in $3 \mathrm{D}$, low bleach rates $(\sim 1.9 \%$ per frame $)$ were chosen to create a $z$-stack with the aim of applying PiMP to the complex 3D subcellular organization of this microtubule network imaged at 1 Airy unit at sub-diffraction resolution (Fig. 3E,F,G), thus demonstrating the 3D potential of PiMP (Fig. 3G).

Given the time needed to acquire a bleaching trail to generate a PiMP image, the strategy is currently not amenable to fast timelapse imaging. However, slowly evolving processes (in the time frame of minutes) could be within reach for live PiMP imaging. We explored this by expressing GFP-tagged doublecortin (DCX), a neuronal microtubule binding protein, in MEFs (Fig. 3H,I,J, quantified in $3 \mathrm{~K})$. We imaged at $2 \mathrm{~Hz}$ over a period of 100 seconds, and assigned 50 consecutive images to each time 

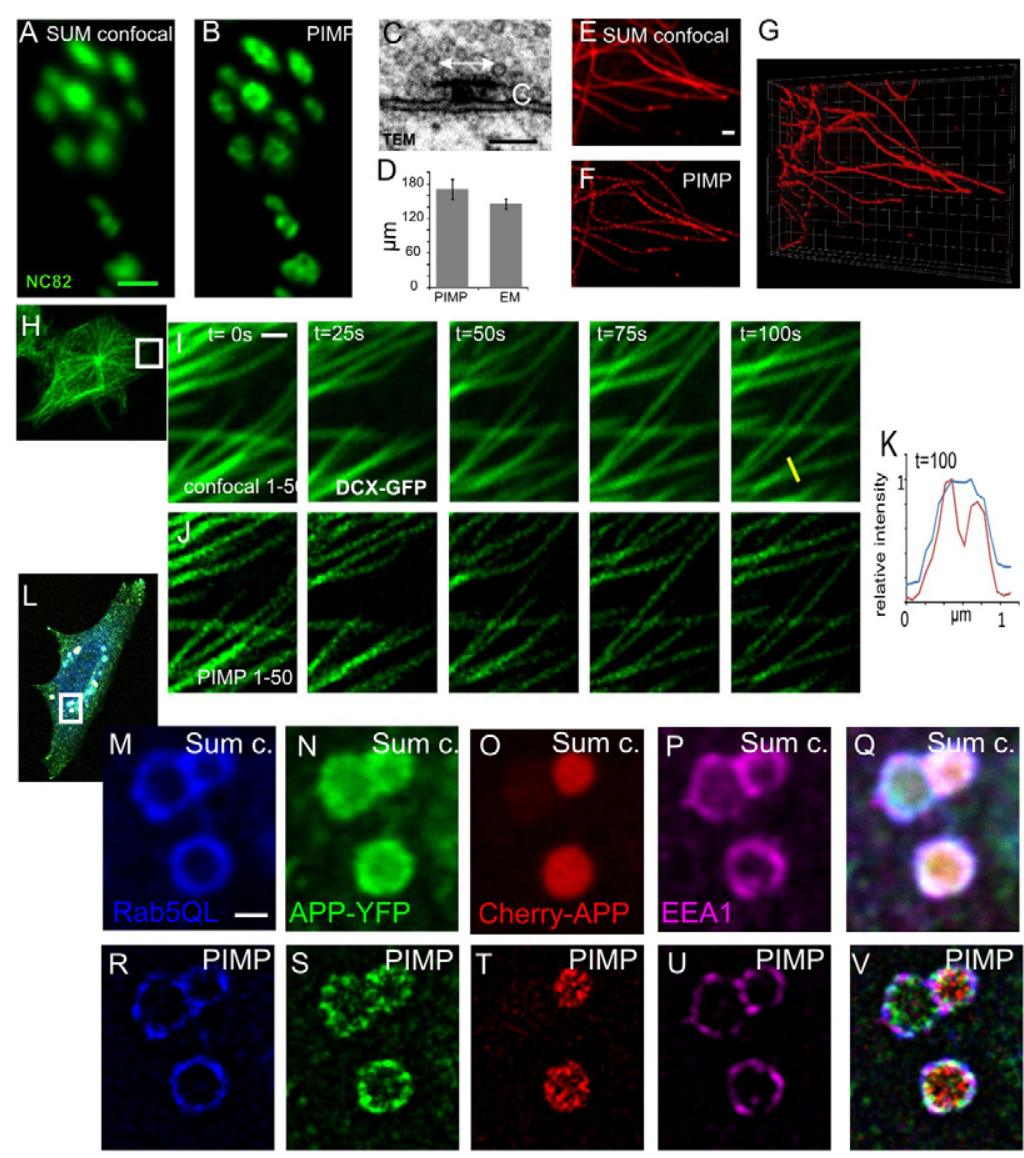

Fig. 3. Standard 3D, live and multicolor PiMP of biological specimens. (A) Confocal scan of an NMJ bouton immunolabeled with monoclonal antibody NC82 recognizing the C-terminal end of Bruchpilot (BRP) and labeling dense bodies or 'T-bars' at synapses. (B) PiMP of the same region. (C) Transmission electron micrograph (TEM) of a single T-bar and (D) quantification of T-bar size in PiMP (hole diameter) and in TEM (arrow in C). Student's $t$-test, not significant. (E) Immunolabeled (Alexa Fluor 568) microtubules in MEF cells. Shown is the sum of the 100 bleach images of a single slice from a stack of eight sections. (F) PiMP processed single slice of E. (G) 3D reconstruction of the stack of eight sections.

(H) Overview of MEF cell expressing a microtubule-binding protein (doublecortin, DCX) fused to GFP. (I) Time course of five times 25 seconds of the box shown in $\mathrm{H}$. At each time point 50 confocal images are summed. (J) The corresponding PiMP image at the respective time points. PiMP discriminates the two tubules better than in the original image. (K) Line graph comparing I with $\mathrm{J}$, at the positions indicated by the yellow line in I. (L) HeLa cells were cotransfected with Cerulean-RAB5(Q79L) and Cherry-APP-YFP. 24 hours later, cells were fixed, immunostained for EEA1 and mounted before imaging. The RAB5 mutant leads to enlarged endosomes, whereas ectodomain shedding of APP leads to the accumulation of Cherry-APP in the lumen of these enlarged endosomes. Overview of the cell is shown in L. White rectangle indicates the area further analyzed in M-V. (M-P) Sum of 50 confocal images (at one $z$ position) and (R-U) the respective PiMP images of (M,R) CeruleanRab5Q79L (blue), (N,S) APP-YFP (green), (O,T) Cherry-APP (red) and (P,U) EEA1 (purple). (Q) Merge of M, N and P. (V) Merge of ( $R, S$ and $U)$. PiMP analysis reveals the distinct microdomain localization of APP-YFP versus CFP-RAB5 and EEA1-positive domains. Scale bars: $1 \mu \mathrm{m}$ for all panels except C, which is $100 \mathrm{~nm}$. Details on imaging conditions are given in supplementary material Table S1. point ( 25 seconds; $\sim 0.3 \%$ bleaching per frame). Within this time frame, not all fluorophores binding the microtubules bleached, and new ones were probably incorporated and used in subsequent time points to generate a bleach series of total 250 images (Henriques et al., 2011; Izeddin et al., 2011). Thus, although our time resolution was in the seconds range, we could monitor the growth of microtubules live and at significantly improved resolution using PiMP. Similar to live PALM imaging (Shroff et al., 2008), the time resolution achievable was largely dependent on imaging speed and on the number of differential images needed to reconstruct the image, thereby avoiding phototoxicity as much as possible. In contrast to PALM imaging, fewer images were required for the reconstruction with PiMP because more information per differential image is used compared with single-molecule techniques.

Finally, we used our method for multicolor imaging of protein localization in subcellular organelles and investigated whether we could discern microdomain or nanodomain preferences. In Alzheimer's disease pathology, the major component of senile plaques consists of $A \beta$ peptides. These short peptide fragments are generated from a larger amyloid precursor protein (APP) by two subsequent proteolytic events mediated through BACE1 and $\gamma$-secretase, respectively (Sannerud and Annaert, 2009). The prevailing idea is that the early endosomes constitute a major site for BACE1-mediated processing of APP (Schneider et al., 2008; Sannerud et al., 2011). This can be demonstrated by coexpressing CFP-RAB5(Q79L) (a dominant active form of RAB5 giving rise to enlarged endosomes) with Cherry-APP-
YFP (a dual fluorescent-tagged APP bearing Cherry in its ectodomain and YFP at its C-terminus) (Sannerud et al., 2011). Confocal analysis of enlarged endosomes clearly shows a luminal build-up of Cherry-APP, representing the shed APP ectodomain, which differs from the localization of the APP C-terminal fragment (represented by APP-YFP) on the limiting membrane. Obviously, as compared with a summed confocal image, PiMP resolves these luminal versus membrane-tethered APP fragments far better (Fig. 3L-V). Interestingly, PiMP additionally reveals that although CFP-RAB5 and endogenous EEA1 co-segregate on the same micro- or nanodomains, APP-YFP is clearly clustered in distinct domains (Fig. 3L-V). Thus, PiMP can reveal the microdomain organization of membranes with much more detail and in a multicolor mode.

Thus far we presented data from imaging with high numerical aperture (NA) lenses. However, PiMP leads to a relative improvement of resolution, as defined by the optical system and thus, potentially, also applies to lenses with low NA. We therefore imaged the synaptic marker syndapin (Kumar et al., 2009) at synaptic boutons of Drosophila neuromuscular junctions (NMJs) using different objective lenses and both a wide-field and a confocal system (Fig. 4). As shown in Fig. 4A,B, and using a 0.45 NA $20 \times$ objective lens, wide-field images of individual boutons $(\sim 2 \mu \mathrm{m}$ in diameter) appear as blurred round structures. Imaging the same area multiple times using the same lens and camera, and processing the data using PiMP strongly increased resolution, allowing us to discern the synaptic areas (Fig. 4C). Next, we used a 1.4 NA $100 \times$ lens and obtained wide-field 

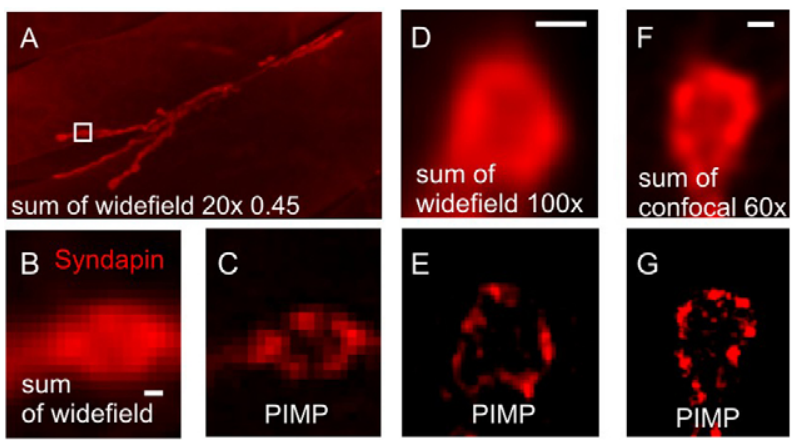

Fig. 4. PiMP imaging in wide-field using low NA lenses. Synaptic boutons of neuromuscular junctions of Drosophila larvae revealed by syndapin antibody staining. (A) Overview of a complete third-instar larval NMJ on muscle 6 and 7. (B) Zoomed sum of 100 wide-field images of a single synaptic bouton. (C) PiMP image of B (using $20 \times 0.45$ NA objective). (D) For comparison, a synaptic bouton of the same sample is imaged with a wide-field set-up using a better resolving lens $(100 \times 1.4 \mathrm{NA})$ as in A-C. (E) Same image using PiMP. (F) Image obtained on a confocal microscope with a $60 \times(1.4$ NA) objective. (G) PiMP version of F. In all cases, PiMP images reveal more detail as compared with the summed images. Scale bars: $1 \mu \mathrm{m}$. Details on imaging conditions are given in supplementary material Table S1.

images of NMJs immunolabeled for syndapin. Although under these conditions the synaptic accumulation of the syndapin is evident (Fig. 4D,E), processing the same wide-field images using PiMP generated significantly more information with syndapin immunoreactivity now clearly concentrated in discrete foci akin to other postsynaptically localized proteins including DLG/ PSD95 (Kumar et al., 2009). We further confirmed these findings using confocal microscopy followed by PiMP (Fig. 4F,G). Our data indicate that PiMP is a generally applicable strategy that can be used in combination with wide-field microscopy, as well as confocal imaging.

\section{Discussion}

PiMP is a novel super-resolution imaging method that allows discriminating features beyond the diffraction barrier. It does so by calculating differential images from a series of bleaching events and subsequently analyzing and enhancing subpopulations of the bleached fluorophores. The method is not limited to bleaching and can also be used for any other stochastic event such as photoconversion or blinking. The nonlinear processing in (and of) the differential images allows for the reconstitution and localization of the underlying structures far better than if all fluorophores are imaged and processed simultaneously (linearly or nonlinearly). In addition to the theoretical background we provide a variety of biological examples to demonstrate the broad applicability of PiMP for multifluorescent imaging, using standard immunolabeling or fluorescent protein tags, 3D reconstruction and (limited) live imaging of slowly evolving processes.

Methods such as PALM and STORM can resolve structures at close distance by sporadic activation of individual fluorophores followed by fitting, assuming that the fluorescence comes from only one point (Huang et al., 2010) or from a small number of separatable fluorophores (Holden et al., 2011; Huang et al., 2011). STED achieves this by turning neighboring fluorophores off using a second donut-shaped STED laser in addition to the excitation laser (Klar et al., 2000). Hence, such methods either localize single molecules, or in the continuous case, use spatially non-uniform illumination and a nonlinear photo-response (Heintzmann and Gustafsson, 2009). PiMP is based on the common principle used in true pointillistic techniques (e.g. PALM and STORM) to improve the resolution by separating emitters in time. However, PiMP differs fundamentally from these localization methods because (1) single-molecule separation is not a requirement and (2) the starting point of PiMP is the complete fluorescently stained sample. It thus circumvents the need for specially tailored fluorophores, media or ground state depletion of the fluorescence in a sample.

Given that PiMP acts beyond the single-molecule regime, it is therefore different from methods that construct the image from absolute positions of individual fluorophores, either through photo-activation, bleaching, blinking, return from excited (dark) or triplet states, including PALM, STORM, ground state depletion (GSDM), single-molecule high-resolution imaging with photobleaching (SHRImP), nanometer-localized multiple single-molecule (NALMS) or single-molecule super-resolution of cellular nanostructures using subtractive images (Gordon et al., 2004; Qu et al., 2004; Ram et al., 2006; Fölling et al., 2008; Kaufmann et al., 2009). PiMP also does not require quantum dots as used in Triexciton imaging with three-dimensional subdiffraction resolution (Hennig et al., 2009).

As the basis for PiMP is the analysis of a time series of bleach events, it is related to the previously described super-resolution optical fluctuation imaging SOFI (Dertinger et al., 2009), which also analyzes the fluctuation behavior of pixels over time to obtain the final reconstruction. SOFI does so by using higher statistical cumulants. In contrast to SOFI, PiMP does not require repeatedly blinking emitters, but can deal with simple fluorophore bleaching. The idea behind PiMP is to perform nonlinear feature-enhancing image processing on sub-images before joining the jigsaw pieces consisting of sub-populations of the object, leading to a much improved brightness linearity when compared with SOFI. The PiMP strategy to combine sub-images highlighting sub-populations of fluorophores is also related to structured illumination (Heintzmann and Benedetti, 2006) and dynamic speckle illumination microscopy (Ventalon et al., 2007). For these techniques, algorithms have been described that do not require the knowledge of the illumination pattern but that allow to achieve super-resolution and improved optical sectioning using non-linear image-processing strategies (Heintzmann and Benedetti, 2006; Ventalon et al., 2007). PiMP uses a similar principle (without the need of upfront knowledge of the structure or the illumination pattern) but uses a stochastic process instead of projecting a random pattern for optical sectioning and superresolution. Therefore unlike non-linear techniques such as nonlinear SIM and STED (Gustafsson, 2005; Heintzmann, 2006; Hell, 2007), PiMP has no necessity for spatially controlled saturated fluorophore depletion, as in STED, or saturated excitation, as in saturated patterned excitation (Heintzmann et al., 2002).

PiMP operates under various imaging modalities and to underline this generality we have shown that PiMP goes beyond the resolution limit as defined by Abbe's diffraction limit of the actual device, be it a wide-field system or a confocal microscope. Thus PiMP also improves the resolution of images acquired with low NA lenses (Fig. 4), further extending the potential implementations of PiMP. The PiMP concept does not rely on a specific image-processing step, as long as individual 
images are processed in a non-linear way. Thus other processing steps could be applied to enhance and/or detect the bleach peaks and maxima of the uneven intensity distributions in the differential images. Such methods could for example be other high-pass filters, the direct detection of local maxima or edge enhancement or even non-linear deconvolution; however, as demonstrated in this work, and importantly for a fast and broad dissemination of the approach, even simple filters yield superresolution.

PiMP performance is directly coupled to the bleach rate and its resolving power is comparable but not fundamentally limited to linear approaches such as structured illumination. PiMP can improve the observed resolution by more than a factor of two [i.e. around $96 \mathrm{~nm}$ using a $1.2 \mathrm{NA}$ lens (high-sensitivity conditions; supplementary material Fig. S3)], similar to commercial STED and SIM, and within an order of magnitude from the resolution that is theoretically obtainable by single-molecule localization methodologies (Huang et al., 2010).

Although it is clear that the above methods are valuable and that PALM, STORM and STED set-ups can exceed the resolution attained by PiMP. The major advantage of PiMP is that it improves resolution with no need for specialized instrumentation, special labels or single-molecule accuracy, allowing for multicolor 3D imaging of subcellular compartments, organelles and macromolecular structures in cells and tissues. This makes PiMP attractive to a rapidly expanding number of cell biologists who require significantly higher resolution in combination with multicolor imaging to address their research questions.

\section{Materials and Methods}

\section{Modelling and theory}

A microscopy image $g(x)$ can be approximated by

$$
g(x)=\int h(\zeta-x) f(\xi) d \xi,
$$

with $h$ being the point spread function (PSF) and $f(x)$ being the object imaged. This convolution is written as

$$
G(k)=H(k) F(k),
$$

in Fourier space with capital letters denoting the respective Fourier-transformed quantities. The optical transfer function $H(k)$ is band limited, the famous Abbe limit, which corresponds to a real space grating constant of $D_{\min }=0.5 \lambda_{e m} / \mathrm{NA}$, with $\lambda_{e m}$ being the wavelength and NA being the numerical aperture of the objective lens. Spatial information of the object beyond the Abbe limit frequency is lost in the image $g$.

Assuming that the object, is constituted by a grid of $n$ irresolvable sub-objects at a distance $d<D_{\min }$, the object can be considered as the sum over all the subobjects. Therefore, imaging the total structure means imaging:

$$
f(x)=\sum f_{j}(x)
$$

and consequently,

$$
G(k)=\sum_{j} H(k) F_{j}(k),
$$

where $f_{j}(x)$ is the function that describes the sub-object $j$.

If we consider imaging such a regular array of points of equal brightness at distances below Abbe's limit, the image would be of uniform brightness. During the stochastic bleaching the brightness fluctuates, which can be analyzed for superresolution. A formal argument against a super-resolution strategy using bleaching is that all images of a bleach series are subject to the same diffraction limit and that therefore no super-resolution information can be retrieved. However, it is important to realize that the original, meaning the object, is decomposed by bleaching [showing stochastic selections of $f_{j}(x)$ ]. The resulting brightness fluctuations will cluster around the positions of the structures harboring the fluorophores in consequent bleach images. This reveals sub-populations of fluorophores allowing retrieval of information on the underlying structure.

The non-linear image processing steps in PiMP sharpen the retrieved information beyond the Abbe limit. Owing to the stochastic nature of bleaching, these high-frequency guesses are on average justified allowing the reconstruction of information beyond the Abbe limit.

Similarly, lower-frequency information caused by higher-frequency sample details enters the pass-band of the microscope. If pictured this way, this is akin to structured illumination (SI) microscopy (Heintzmann, 2006). Thus, PiMP shares some similarity to super-resolution structured illumination approaches with random patterns (Heintzmann and Benedetti, 2006; Ventalon et al., 2007), by decomposing the object, more structural information of the object can be retrieved. This is no violation to equation (1) or equation (2) because of the linearity of the imaging, the sum of the sub-images of all $f_{\mathrm{j}}(x)$ will be equal to equation (3).

To recover a sufficient amount of information, several bleached images need to be acquired, i.e. in a homogeneously labeled object, maxima will be detected during bleaching at uniformly distributed positions throughout the structure. Alternatively, in the case of a detailed structure, the maxima will be detected at similar (meaning re-occurring) positions, yielding a re-constructed object. This obviously requires some degree of redundancy in the labeling. To see how reasonable that redundancy is, we have to consider a typical biological preparation, the structural information to be retrieved and standard labeling and expression protocols. Therefore, in the following, we consider as examples the protein Brp and microtubules as shown in the main text. It is expected that 25 Brp molecules form a T-bar. That means that primary antibodies bind those antigens with high specificity. Next, polyclonal secondary antibodies are used. Multiple secondary antibodies can bind one primary antibody. The secondary antibodies are commercially available antibodies that are labeled with multiple fluorophores. According to our information (Invitrogen Manuals; IgG Alexa whole antibody conjugates holding 2-8 fluorophores), five fluorophores is a reasonable estimate for a standard secondary antibody. Consequently we estimate 250 fluorophores per T-bar. For microtubules, approximately 13 proto-filaments form a quasi-helical cylinder ( $25 \mathrm{~nm}$ width) with one turn being of $4 \mathrm{~nm}$ length (Pollard and Earnshaw, 2002). This means that the structural information is $55 \mathrm{~nm}[25 \mathrm{~nm}$ plus twice the dual antibody length $(2 \times 15 \mathrm{~nm})]$. That is roughly 13 turns resulting in 169 ( $\alpha$ and $\beta$ ) antigens. If about half of them are available because of microtubule binding proteins, we can expect up to $\sim 840$ fluorophores. If we use a GFP-conjugated microtubule-binding protein (e.g. doublecortin), we can expect up to 84 of them. In the simulations, we have used between 50 and 100 fluorophores. From the way the biological samples are prepared, we can see that the assumption of clusters of fluorophores is reasonable.

\section{Simulation of data}

Simulations were performed using Matlab 2008b (Mathworks) and the Matlab toolbox Dipimage 2.3 (TU Delft). First, an object (50 nm pixel pitch) was generated in silico. The object was labeled with dye molecules according to the local concentration per pixel. For simplicity, we assumed each molecule had uniform brightness, even though this is not a requirement for PiMP. A bleach series was then generated by successively removing dye molecules in each frame. Each molecule was individually tested in each frame whether it bleached according to the given bleach probability of a single molecule per frame, $P_{\text {bleach. }}$ A list of the molecules remaining after each frame was kept during the time course of the simulation forming the basis for testing of bleaching in the next frame. A theoretical PSF (1.2 NA; $520 \mathrm{~nm}$ emission light and a refractive index of the sample of 1.33) was computed using vectorial theory and convolved with the bleach series to obtain the corresponding ideal images. These images were subjected to Poisson-distributed photon noise with the number of photons per molecule as indicated to yield the simulated detection images.

To calculate the probabilities for Fig. 1G, a matrix of probabilities for two draws with a starting value of 50 and a probability of $5 \%, 10 \%$ and $20 \%$ was calculated for the range of events from zero to 15 using the Binomial density function in Matlab. Values above 15 were omitted because the probability for these events became smaller and smaller. The probabilities for equal brightness (symmetrical bleaching) and close to symmetrical events based on the similarity of the brightness (difference $<30 \%$ and $<50 \%$ ) was summed.

\section{Data processing}

Pixel-by-pixel image processing was carried out using custom-made routines in Matlab and ImageJ according to the schemes described in the main text. Background correction was applied (where necessary) by subtracting a background value, respectively. Where required, sequential images were also corrected for drift artifacts using the Stackreg plug-in (Thévenaz et al., 1998) or using a correlation function in Matlab. Processing was performed as described in the results section.

\section{Fluorescence imaging}

$0.1 \mu \mathrm{m}$ microspheres (beads) (supplementary material Fig. S5) were imaged using a Radiance 2100 confocal microscope (Zeiss, Jena, Germany), equipped with a 
Nikon 1.4 NA oil-immersion lens. Images in Fig. 2A-D were acquired using a breadboard setup that was described recently (Adam et al., 2011). Its main constituents are $405 \mathrm{~nm}, 488 \mathrm{~nm}, 561 \mathrm{~nm}$ lasers (Spectra Physics, Santa Clara CA) an Olympus (Tokyo, Japan) inverted microscope (IX71 with a 3.3 tube lens), a $100 \times 1.3$ NA oil-immersion lens and a Hamamatsu (Hamamatsu, Japan) ImagEM $512 \times 512$ camera. N-SIM imaging in Fig 2E,F was acquired using a TiE inverted microscope (Nikon, Tokyo, Japan) equipped with a structured illumination unit, a 1.49 NA oil-immersion objective lens and an Andor (Belfast, UK) iXion DU897 EMCCD camera and comprising $488 \mathrm{~nm}$ and $568 \mathrm{~nm}$ laser modules.

Imaging in Fig. 3A,B, Fig. 3H-J and Fig. 4F,G was performed using a Nikon A1R confocal unit mounted on a Ti2000 inverted microscope equipped with a Plan Apo $60 \times$ NA 1.4 oil-immersion lens (Nikon). Fig. 3E-G,L-V were acquired using a Leica (Wetzlar, Germany) SP5 confocal scan head mounted on a DM $6000 \mathrm{CS}$ upright Leica microscope fitted with a $63 \times 1.4$ NA oil-immersion lens. In Fig. 4A,B,C, a wide-field system with a 0.45 NA $20 \times$ lens was used (IN Cell Analyzer, GE Healthcare, Chalfont St Giles, UK). In Fig. 4D,E, a Zeiss upright microscope equipped with a $63 \times 1.4 \mathrm{NA}$ oil-immersion lens was used. The Qimaging QICAM camera (Surrey, BC, Canada) and shutter was driven by $\mu$ Manager software (Edelstein et al., 2010). Samples were sequentially imaged using imaging conditions listed in supplementary material Table S1.

\section{Sample preparation}

$0.1 \mu \mathrm{m}$ TetraSpeck microspheres (beads) (Invitrogen, Carslbad, CA) were dispersed on a coverslip and imaged using $543 \mathrm{~nm}$ laser line was used for excitation and $600 \mathrm{~nm}$ long pass for emission. Mouse embryonic fibroblasts (MEFs) were grown in DMEM/F12 supplemented with $10 \%$ FCS, fixed with $4 \%$ paraformaldehyde in PBS and processed for indirect immunofluorescence (Spasic et al., 2007).

Microtubules in MEFs were immunolabeled using monoclonal antibodies against $\alpha$-tubulin (mAb DMIA, Sigma) followed by Alexa-Fluor-488- and AlexaFluor-568-conjugated goat anti-mouse secondary antibodies (Invitrogen). Live MEFs were transfected with doublecortin-GFP (gift from Reinhard Köster, Helmholtz Zentrum München Germany) with Lipofectamine 2000 (Invitrogen) according to the manufacturer's instructions. For dSTORM imaging, cells were grown on Lab-Tek chambered coverglass systems. Before imaging, cysteamine (Sigma) was added according to published methods (Heilemann et al., 2009).

Third-instar wild-type Drosophila larvae were fixed and processed for immunostaining as described previously (Fouquet et al., 2009). To reveal Cterminal BRP, the mAb NC82 (Developmental Studies Hybridoma Bank, Iowa) primary and Alexa Fluor 488 (Invitrogen) secondary antibodies were used. Guinea pig syndapin antibody was a gift from Hugo Bellen (HHMI - Baylor College of Medicine, Houston, TX) generated against full-length recombinant protein and was combined with Alexa Fluor 647 (Invitrogen) antibody (see supplementary material Table S1).

HeLa cells (CCL2 clone) were transiently transfected with Rab5(Q79L) (obtained from Marino Zerial, Max Planck Institute, Dresden, Germany). Double tagged Cherry-APP-YFP was previously described (Sannerud et al., 2011). HeLa cells were transfected with FugeneHD (Promega). Rabbit anti-early endosomal antigen 1 (EEA1) antibody was purchased from Sigma and was revealed with Alexa-Fluor-647-conjugated secondary antibody (Invitrogen).

\section{Transmission electron microscopy}

Dissected third-instar wild-type Drosophila larvae were processed for TEM as described previously (Kasprowicz et al., 2008) and electron micrographs of synaptic boutons and T-bars were obtained on a Jeol (Tokyo, Japan) 2100 EM microscope operated at $200 \mathrm{kV}$. T-bar size was measured as the 'table-top' length of $19 \mathrm{~T}$ bars taken from three animals. Ring size in PiMP (Fig. 3D) was measured as inner ring diameter on 19 samples.

\section{Acknowledgements}

We thank Johan Hofkens and Jonas Ries for discussions, Stephan Sigrist, Hugo Bellen and Reinhard Köster for reagents, Pieter Baatsen (VIB/KULeuven EM core facility) for help with TEM, and Susana Rocha (Division of Molecular and Nano Materials, Department of Chemistry, KULeuven) for support on the dSTORM measurements. The authors would also like to acknowledge the Nikon Imaging center at Institute Curie CNRS Paris and the help of David Pointu and Philippe Baert for the acquisition of the N-SIM images.

\section{Funding}

This work was supported by Vlaams Instituut voor Biotechnologie (VIB), KULeuven and a Methusalem grant, as well as grants from the Interuniversity Attraction Poles (IAP) [grant number P6/43 to
W.A.]; the Hercules Foundation [grant numbers AKUL/09/037 and AKUL058 (HER/08/021) to W.A.]; Fonds Wetenschappelijk Onderzoek - Vlaanderen (FWO) [grant numbers G.0663.09 and G.0.754.10.N to W.A. and G.0747.09, G094011 and G095511 to P.V.]; the Alzheimer Association [grant numbers IIRG-08-91535 and SAO-FRMA-2010 to W.A.]; the Francqui Foundation; the Marie Curie Foundation [grant number MEXT-CT-2006-042267 to P.V. and FP7-PIIF-GA-2009-252375 to S.A.M.]; and a European Research Council (ERC) Starting Grant [grant number 260678 to P.V.].

Supplementary material available online at http://jcs.biologists.org/lookup/suppl/doi:10.1242/jcs.098939/-/DC1

\section{References}

Abbe, E. (1873). Contributions to the theory of the microscope and the microscopic perception (translated from German). Arch. Mikrosk. Anat. 9, 413-468.

Adam, V., Moeyaert, B., David, C. C., Mizuno, H., Lelimousin, M., Dedecker, P., Ando, R., Miyawaki, A., Michiels, J., Engelborghs, Y. et al. (2011). Rational design of photoconvertible and biphotochromic fluorescent proteins for advanced microscopy applications. Chem. Biol. 18, 1241-1251.

Betzig, E., Patterson, G. H., Sougrat, R., Lindwasser, O. W., Olenych, S. Bonifacino, J. S., Davidson, M. W., Lippincott-Schwartz, J. and Hess, H. F. (2006). Imaging intracellular fluorescent proteins at nanometer resolution. Science 313, 1642-1645.

Dertinger, T., Colyer, R., Iyer, G., Weiss, S. and Enderlein, J. (2009). Fast, background-free, 3D super-resolution optical fluctuation imaging (SOFI). Proc. Natl. Acad. Sci. USA 106, 22287-22292.

Edelstein, A., Amodaj, N., Hoover, K., Vale, R. and Stuurman, N. (2010). Computer control of microscopes using microManager. Curr. Protoc. Mol. Biol. 14, 20.

Fölling, J., Bossi, M., Bock, H., Medda, R., Wurm, C. A., Hein, B., Jakobs, S., Eggeling, C. and Hell, S. W. (2008). Fluorescence nanoscopy by ground-state depletion and single-molecule return. Nat. Methods 5, 943-945.

Fouquet, W., Owald, D., Wichmann, C., Mertel, S., Depner, H., Dyba, M., Hallermann, S., Kittel, R. J., Eimer, S. and Sigrist, S. J. (2009). Maturation of active zone assembly by Drosophila Bruchpilot. J. Cell Biol. 186, 129-145.

Gong, W., Si, K., Chen, N. and Sheppard, C. J. (2010). Focal modulation microscopy with annular apertures: a numerical study. J. Biophotonics 3, 476-484.

Gordon, M. P., Ha, T. and Selvin, P. R. (2004). Single-molecule high-resolution imaging with photobleaching. Proc. Natl. Acad. Sci. USA 101, 6462-6465.

Gustafsson, M. G. (2000). Surpassing the lateral resolution limit by a factor of two using structured illumination microscopy. J. Microsc. 198, 82-87.

Gustafsson, M. G. (2005). Nonlinear structured-illumination microscopy: wide-field fluorescence imaging with theoretically unlimited resolution. Proc. Natl. Acad. Sci. USA 102, 13081-13086.

Gustafsson, M. G., Agard, D. A. and Sedat, J. W. (1999). I5M: 3D widefield light microscopy with better than $100 \mathrm{~nm}$ axial resolution. J. Microsc. 195, 10-16.

Hallermann, S., Kittel, R. J., Wichmann, C., Weyhersmüller, A., Fouquet, W., Mertel, S., Owald, D., Eimer, S., Depner, H., Schwärzel, M. et al. (2010). Naked dense bodies provoke depression. J. Neurosci. 30, 14340-14345.

Heilemann, M., van de Linde, S., Schüttpelz, M., Kasper, R., Seefeldt, B., Mukherjee, A., Tinnefeld, P. and Sauer, M. (2008). Subdiffraction-resolution fluorescence imaging with conventional fluorescent probes. Angew. Chem. Int. Ed. Engl. 47, 6172-6176.

Heilemann, M., van de Linde, S., Mukherjee, A. and Sauer, M. (2009). Superresolution imaging with small organic fluorophores. Angew. Chem. Int. Ed. Engl. 48, 6903-6908.

Heintzmann, R. (2006). Handbook of Biological Confocal Microscopy. New York: Springer.

Heintzmann, R. and Benedetti, P. A. (2006). High-resolution image reconstruction in fluorescence microscopy with patterned excitation. Appl. Opt. 45, 5037-5045.

Heintzmann, R. and Gustafsson, M. G. (2009). Subdiffraction resolution in continuous samples. Nat. Photonics 3, 362-364.

Heintzmann, R., Jovin, T. M. and Cremer, C. (2002). Saturated patterned excitation microscopy-a concept for optical resolution improvement. J. Opt. Soc. Am. A Opt. Image Sci. Vis. 19, 1599-1609.

Hell, S. W. (2007). Far-field optical nanoscopy. Science 316, 1153-1158.

Hell, S. W. and Wichmann, J. (1994). Breaking the diffraction resolution limit by stimulated emission: stimulated-emission-depletion fluorescence microscopy. Opt. Lett. 19, 780-782.

Hennig, S., van de Linde, S., Heilemann, M. and Sauer, M. (2009). Quantum dot triexciton imaging with three-dimensional subdiffraction resolution. Nano Lett. 9, 2466-2470.

Henriques, R., Lelek, M., Fornasiero, E. F., Valtorta, F., Zimmer, C. and Mhlanga, M. M. (2010). QuickPALM: 3D real-time photoactivation nanoscopy image processing in ImageJ. Nat. Methods 7, 339-340.

Henriques, R., Griffiths, C., Hesper Rego, E. and Mhlanga, M. M. (2011). PALM and STORM: unlocking live-cell super-resolution. Biopolymers 95, 322-331. 
Hess, S. T., Girirajan, T. P. and Mason, M. D. (2006). Ultra-high resolution imaging by fluorescence photoactivation localization microscopy. Biophys. J. 91, 4258-4272.

Holden, S. J., Uphoff, S. and Kapanidis, A. N. (2011). DAOSTORM: an algorithm for high- density super-resolution microscopy. Nat. Methods 8, 279-280.

Huang, B., Babcock, H. and Zhuang, X. (2010). Breaking the diffraction barrier: super-resolution imaging of cells. Cell 143, 1047-1058.

Huang, F., Schwartz, S. L., Byars, J. M. and Lidke, K. A. (2011). Simultaneous multiple-emitter fitting for single molecule super-resolution imaging. Biomed. Opt. Express 2, 1377-1393.

Izeddin, I., Specht, C. G., Lelek, M., Darzacq, X., Triller, A., Zimmer, C. and Dahan, M. (2011). Super-resolution dynamic imaging of dendritic spines using a low-affinity photoconvertible actin probe. PLOS ONE 6, e15611.

Kasprowicz, J., Kuenen, S., Miskiewicz, K., Habets, R. L., Smitz, L. and Verstreken, P. (2008). Inactivation of clathrin heavy chain inhibits synaptic recycling but allows bulk membrane uptake. J. Cell Biol. 182, 1007-1016.

Kaufmann, R., Lemmer, P., Gunkel, M., Weiland, Y., Muller, P., Hausmann, M., Baddeley, D., Amberger, R. and Cremer, C. (2009). SPDM - Single Molecule Superresolution of Cellular Nanostructures. In Proceedings of SPIE, vol. 7185, pp. 71850J. San Jose, CA, USA International Society for Optical Engineering.

Kittel, R. J., Wichmann, C., Rasse, T. M., Fouquet, W., Schmidt, M., Schmid, A., Wagh, D. A., Pawlu, C., Kellner, R. R., Willig, K. I. et al. (2006). Bruchpilot promotes active zone assembly, $\mathrm{Ca} 2+$ channel clustering, and vesicle release. Science 312, 1051-1054.

Klar, T. A., Jakobs, S., Dyba, M., Egner, A. and Hell, S. W. (2000). Fluorescence microscopy with diffraction resolution barrier broken by stimulated emission. Proc. Natl. Acad. Sci. USA 97, 8206-8210.

Kumar, V., Alla, S. R., Krishnan, K. S. and Ramaswami, M. (2009). Syndapin is dispensable for synaptic vesicle endocytosis at the Drosophila larval neuromuscular junction. Mol. Cell. Neurosci. 40, 234-241.

Pollard, T. D. and Earnshaw, C. W. (2002). Cell Biology. Philadelphia: Saunders.

Qu, X., Wu, D., Mets, L. and Scherer, N. F. (2004). Nanometer-localized multiple singlemolecule fluorescence microscopy. Proc. Natl. Acad. Sci. USA 101, 11298-11303.
Ram, S., Ward, E. S. and Ober, R. J. (2006). Beyond Rayleigh's criterion: a resolution measure with application to single-molecule microscopy. Proc. Natl. Acad. Sci. USA 103, 4457-4462.

Rust, M. J., Bates, M. and Zhuang, X. (2006). Sub-diffraction-limit imaging by stochastic optical reconstruction microscopy (STORM). Nat. Methods 3, 793796.

Sannerud, R. and Annaert, W. (2009). Trafficking, a key player in regulated intramembrane proteolysis. Semin. Cell Dev. Biol. 20, 183-190.

Sannerud, R., Declerck, I., Peric, A., Raemaekers, T., Menendez, G., Zhou, L., Veerle, B., Coen, K., Munck, S., De Strooper, B. et al. (2011). ADP ribosylation factor 6 (ARF6) controls amyloid precursor protein (APP) processing by mediating the endosomal sorting of BACE1. Proc. Natl. Acad. Sci. USA 108, E559E568.

Schneider, A., Rajendran, L., Honsho, M., Gralle, M., Donnert, G., Wouters, F., Hell, S. W. and Simons, M. (2008). Flotillin-dependent clustering of the amyloid precursor protein regulates its endocytosis and amyloidogenic processing in neurons. J. Neurosci. 28, 2874-2882.

Shroff, H., Galbraith, C. G., Galbraith, J. A. and Betzig, E. (2008). Live-cell photoactivated localization microscopy of nanoscale adhesion dynamics. Nat. Methods 5, 417-423.

Spasic, D., Raemaekers, T., Dillen, K., Declerck, I., Baert, V., Serneels, L. Füllekrug, J. and Annaert, W. (2007). Rerlp competes with APH-1 for binding to nicastrin and regulates gamma-secretase complex assembly in the early secretory pathway. J. Cell Biol. 176, 629-640.

Thévenaz, P., Ruttimann, U. E. and Unser, M. (1998). A pyramid approach to subpixel registration based on intensity. IEEE Trans. Image Process. 7, 27-41.

van de Linde, S., Krstix, I., Prisner, T., Doose, S., Heilemann, M. and Sauer, M. (2011). Photoinduced formation of reversible dye radicals and their impact on superresolution imaging. Photochem. Photobiol. Sci. 10, 499-506.

Ventalon, C., Heintzmann, R. and Mertz, J. (2007). Dynamic speckle illumination microscopy with wavelet prefiltering. Opt. Lett. 32, 1417-1419. 